\title{
Does the present moment depend on the moments not lived?
}

\author{
Commentary on Merker, B., Williford, K., \& Rudrauf, D. (2021), The Integrated Information Theory of \\ consciousness: A case of mistaken identity. Behavioral and Brain Sciences.
}

Romain Brette

Sorbonne Université, INSERM, CNRS, Institut de la Vision

17 rue Moreau, F-75012 Paris, France

+33153462536

romain.brette@inserm.fr

http://romainbrette.fr

\begin{abstract}
Integrated Information Theory postulates that a conscious experience depends on a repertoire of hypothetical experiences (the axiom of information). This makes consciousness depend on the context that constrains the set of possibilities and on the scenarios imagined by the external observer, and not only on the system itself.
\end{abstract}

\section{Main text}

"Information is one of the most confused notions in contemporary intellectual life", Searle once remarked (Searle, 2013). Merker et al. (Merker et al., 2021, p21) rightfully point out that Integrated Information Theory (IIT) conflates informativeness in the ordinary sense (a semantic notion relative to a cognitive agent) with information capacity as defined by Shannon (a formal syntactic notion defined by the observer). A similar confusion is at play in the literature about "neural codes" (Brette, 2019a).

Consider one of IIT's "immediately evident" claims: "an experience of pure darkness is what it is by differing [...] from [...] other possible experiences" (Oizumi et al., 2014, p2). Superficially, the claim has some intuitive appeal: we appreciate the particular quality of darkness because we can compare it to different experiences we have had before, e.g. lightness. But the formalization of IIT makes it clear that "possible experiences" refer neither to memory nor to anticipation by the system, but to formal possibilities defined by the external observer, alternative realities that the observer envisions but are not actually realized. Is it immediately evident that our present experience depends on hypothetical experiences that we have never had or even imagined?

To see the difficulty with this proposition, consider the photodiode consisting of a sensor and a detector, which Tononi considers as minimally conscious (Tononi, 2008); in a later version of IIT, the photodiode is only conscious when the detector feeds back onto the sensor (Oizumi et al., 2014), but this makes no difference to the present discussion. Observing the state of the detector reduces the uncertainty about the previous state of the system by one bit, making the mechanism minimally conscious. This uncertainty reduction only occurs because we consider that the photodiode lives in a universe where dark and light exist with equal probabilities. But what if the photodiode actually lived in a sealed opaque box so that light is not a "possible experience"? In this context, the uncertainty reduction is exactly zero, since we know the system is always in the dark state. We therefore conclude that the photodiode is not conscious if it is permanently enclosed in a box, but conscious if it is not. We must also conclude that the photodiode is conscious if it is in a box that someone might remove, even if that event does not actually occur. Thus, consciousness as defined by IIT is not an intrinsic property of the system, contrary to previous claims (Tononi, 2008, p220).

Or are we to consider that, even in the box, the lighted state is still "theoretically" possible given the design of the mechanism? But if we are allowed to count events that can never happen as possible experiences, then where do we stop? We conclude that the repertoire of possible events is defined by the imagination of the observer, and if different minds can imagine different repertoires, then this makes the definition incoherent. In any case, consciousness defined in this way is not an intrinsic property of the system. Even in the framework of property dualism (matter has conscious properties distinct from its physical properties, but linked to them by additional laws), experiences are not 
allowed to depend on scenarios living in the imagination of someone else, but only on the current state or ongoing processes of the system.

Defining consciousness as a function of hypothetical possibilities leads IIT proponents to conclude that inactive systems can be conscious, but only if they can become active again (Oizumi et al., 2014). Specifically, Tononi imagines that a grey stimulus that would not activate color neurons would be perceived as grey, while lesioning the color area would result in a loss of color experience, because the neurons cannot become active again (Tononi, 2015). But what if the experimenter decides instead to cool the color area, a reversible operation? Would the person still perceive color in that case? If the experimenter decided to cool that area permanently, would the person lose color perception from the moment the brain is cooled down? And what happens if the cooling apparatus breaks down and the brain recovers? Does imagining this possibility make the person perceive color all along?

The proposition that an inactive system can be conscious, perhaps in some sort of meditative state (Oizumi et al., 2014, p17), is also questionable. The system cannot itself distinguish between the experience of being inactive (or in any constant state) for a duration A and the same inactivity for a duration $\mathrm{B}$, for that discrimination should be realized as different states. Therefore, the experience of being inactive for any amount of time must be the same as the experience of being inactive for no time at all: a non-existent experience. This is precisely what happens in the TV series "Bewitched" (Brette, 2019b): Samantha the housewife is actually a witch: when she twitches her nose, people freeze; when she twitches it again, they unfreeze and go on as if nothing happened between the two instants. Their brain state was unchanged, their brain processes were stalled, so that they did not experience anything at all. IIT breaks with TV series wisdom because it associates conscious experiences to a state of the system, rather than to its ongoing processes. But to experience is something that a system does something lived, not some formal characteristic of the system's state.

\section{Conflict of interest statement}

The author declares no conflict of interest.

\section{Funding statement}

This work was supported by Agence Nationale de la Recherche (ANR-20-CE30-0025-01), Programme Investissements d'Avenir IHU FOReSIGHT (ANR-18-IAHU-01) and Fondation Pour l'Audition (FPA RD2017-2).

\section{References}

Brette, R. (2019a). Is coding a relevant metaphor for the brain? The Behavioral and Brain Sciences, 144. https://doi.org/10.1017/S0140525X19000049

Brette, R. (2019b). Neural coding: The bureaucratic model of the brain. The Behavioral and Brain Sciences, 42, e243. https://doi.org/10.1017/S0140525X19001997

Merker, B., Williford, K., \& Rudrauf, D. (2021). The Integrated Information Theory of consciousness: A case of mistaken identity. The Behavioral and Brain Sciences, 1-72.

https://doi.org/10.1017/S0140525X21000881

Oizumi, M., Albantakis, L., \& Tononi, G. (2014). From the phenomenology to the mechanisms of consciousness: Integrated Information Theory 3.0. PLoS Computational Biology, 10(5), e1003588. https://doi.org/10.1371/journal.pcbi.1003588

Searle, J. R. (2013, January 10). Can Information Theory Explain Consciousness? The New York Review of Books. http://www.nybooks.com/articles/2013/01/10/can-information-theory-explainconsciousness/

Tononi, G. (2008). Consciousness as integrated information: A provisional manifesto. The Biological Bulletin, 215(3), 216-242. https://doi.org/10.2307/25470707

Tononi, G. (2015). Integrated information theory. Scholarpedia, 10(1), 4164. https://doi.org/10.4249/scholarpedia.4164 